\title{
Pulmonary artery catheters in cardiovascular surgery
}

Ian R. Thomson MD

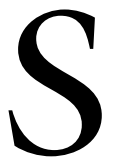

INCE the introduction of the balloon-tipped, pulmonary artery catheter (PAC) in 1970, the PAC has been widely used in patients undergoing cardiovascular surgery. Cardiovascular anesthesiologists initially embraced the PAC with enthusiasm and many used it routinely in patients undergoing coronary artery bypass grafting $(\mathrm{CABG})$ or abdominal aortic reconstruction (AAR). They assumed that by measuring variables that were not available on routine clinical assessment (pulmonary artery occlusion pressure, cardiac output and mixed venous oxygen saturation) perioperative management would change in ways that would improve patient outcome. However, the love affair between cardiovascular anesthesiologists and the PAC, although prolonged and intense, appears to be cooling. This impression is confirmed by the results of a survey conducted by Jacka $e t a l$. and published in this issue of the Journal. ${ }^{1}$

Jacka et al. surveyed 345 anesthesiologists, at 29 different centres in Canada and the United States, during the first four months of 1998. Of 265 responders $(76.8 \%), 214$ delivered at least one anesthetic per month for a cardiac or vascular surgical procedure. The responses of those 214 "cardiovascular anesthesiologists" form the subject of the current publication. Respondents were asked to assess the appropriateness of PAC use in various clinical scenarios involving patients undergoing elective CABG or AAR. Appropriateness was rated on a nine-point scale ranging from 1 (completely inappropriate) to 9 (completely appropriate). Responses were grouped as inappropriate (1-3), uncertain (4-6) and appropriate (7-9). Only $19 \%$ of responders regarded PAC use as appropriate for the usual patient with stable angina and normal ventricular function undergoing either CABG or AAR. However, there was agreement that PAC use was appropriate in certain high-risk scenarios. Seventy-four percent of respondents felt that PAC use was appropriate for patients with impaired left ventric- ular (LV) function (LV ejection fraction $<0.4)$ and $87 \%$ considered PAC use appropriate for those with both unstable angina and impaired LV function.

We can only speculate as to why cardiovascular anesthesiologists have become disenchanted with routine PAC use. The most obvious explanation is that after 30 years of experience there is no evidence from large, randomized controlled trials (RCTs) that PAC use improves outcome in patients undergoing cardiovascular surgery. In patients undergoing CABG, the best available evidence indicates that PAC use does not alter outcome, but may increase resource utilization. ${ }^{2} \mathrm{~A}$ retrospective study observed that low-risk patients undergoing CABG with only central venous pressure (CVP) monitoring had a good outcome. ${ }^{3}$ One large, retrospective, cohort study found that PAC use was associated with increased mortality and resource utilization in patients undergoing CABG. ${ }^{4}$ In low-risk patients undergoing AAR, three small RCTs indicate no improvement in outcome associated with PAC use compared to CVP monitoring. ${ }^{5-7}$ In two of these studies, ${ }^{5,6}$ no protocol for using PAC data to guide therapy was provided, while in the third the PAC was used to guide therapy aimed at achieving specific hemodynamic goals. ${ }^{7}$ In two other small RCTs of patients undergoing elective vascular surgery (some undergoing AAR) the PAC was used to guide therapy aimed at "optimizing" oxygen delivery. ${ }^{8,9}$ These studies also showed no improvement in outcome associated with the PAC. Cardiovascular anesthesiologists may also have been swayed by the results of uncontrolled, observational studies in which PAC use has been associated with increased mortality and resource utilization in various patient populations including those with complicated myocardial infarction, ${ }^{10}$ critical illness, ${ }^{11}$ or undergoing noncardiac surgery. ${ }^{12}$ In the most publicized of these latter studies, the risk of increased mortality related to PAC use, in a mixed population of patients receiving care in an Intensive Care Unit setting, was greatest in

From the Department of Anesthesia, University of Manitoba, Winnipeg, Manitoba, Canada.

Address correspondence to: Dr. I.R. Thomson, Department of Anesthesia, St. Boniface General Hospital, 409 Taché Avenue, Winnipeg, Manitoba R2H 2A6, Canada. Phone: 204-235-3456; Fax: 204-237-3735; E-mail: ithomson@sbrc.ca 
postoperative patients after major surgery. ${ }^{11}$ An alternative explanation is that resource constraints have forced practitioners to use resources and time more economically. This may have led to an accelerated decrease in PAC use. Clinical experience of good outcome in patients not monitored with PACs may also have influenced clinicians' attitudes. Finally, competing technologies such as transesophageal echocardiography (TEE) may be supplanting PAC use in clinical practice. While being arguably less invasive, TEE may give more objective information about preload and ventricular function than PAC monitoring. ${ }^{13}$ It is likely that Jacka et al. also considered this latter explanation, since they sent questionnaires to anesthesiologists from selected institutions "based on known differences in the availability of TEE." Unfortunately, the effect of TEE availability on appropriateness scores is not addressed in the results.

The paper by Jacka et al. makes interesting reading for a number of reasons. First, in the absence of high quality evidence, the opinions of one's peers constitute a useful guide to good practice. Second, the survey results may also indicate appropriate areas for future research. In this regard, it has been suggested that clinical trials are indicated when a state of "clinical equipoise" exists. ${ }^{14}$ Equipoise exists when a significant proportion of clinicians, usually 30-70\%, are uncertain regarding the potential utility of a specific therapy or device. When equipoise does not exist, clinical trials might be unethical. In this regard, Jacka et al. suggest that clinical trials of PAC utility may be unethical in the usual patient, with stable angina and LV ejection fraction 0.4 undergoing either $\mathrm{CABG}$ or AAR, because their survey suggests the absence of equipoise. The mean appropriateness rating for PAC use in this scenario was 3.81, whereas the range for clinical equipoise employed by Jacka et al. was a mean score of 4.0-6.0. Similar objections might be raised to the conduct of clinical trials in patients with LV dysfunction (mean appropriateness score $=7.19$ ).

Several major consensus statements have assessed the benefit of PAC use in cardiovascular surgery and made recommendations regarding the need for further clinical trials. ${ }^{15-17}$ It is interesting that expert opinion from these conferences is not uniformly in accordance with the findings of Jacka et al. Participants at the Pulmonary Artery Catheter Consensus Conference concluded that PAC use was not indicated in low-risk patients undergoing $\mathrm{CABG}$, although the evidence for this was categorized as Grade C (supported by small randomized trials with uncertain results). ${ }^{15}$ In contrast, the same group of experts concluded that the benefit of PAC use in high-risk patients undergoing $\mathrm{CABG}$ was uncertain (also based on Grade $\mathrm{C}$ evidence) and they recom- mended clinical trials in this population. Recently, the Pulmonary Artery Catheterization and Clinical Outcomes (PACCO) workshop, conducted by the National Heart, Lung and Blood Institute and the Food and Drug Administration, identified "low-risk CABG surgery" as one of three high priority areas for future randomized, controlled clinical trials of PAC utility. ${ }^{16}$ Finally, the American Society of Anesthesiologists (ASA) Task Force on Pulmonary Artery Catheterization concluded that "future randomized controlled trials [of PAC utility] should use low-risk patients (e.g., ejection fraction greater than 50\%) and should restrict the analysis to a specific type of surgery (e.g., CABG surgery)". ${ }^{17}$ These disparate conclusions, reached by different groups of experts, and by the survey of Jacka et al., reflect the extent of controversy and lack of good evidence regarding the utility of PACs in cardiovascular surgery.

Should the evidence provided by Jacka et al. lead us to abandon, as unethical, the idea of conducting large, randomized, controlled trials of PAC utility in low-risk patients undergoing cardiovascular surgery? For several reasons, I think not. First, the number of survey respondents was quite small, and the results may not adequately reflect the opinions of the entire population of North American cardiovascular anesthesiologists. Based on caseload, some respondents may have been relatively inexperienced in cardiovascular anesthesia. Second, respondents differed in their opinions with respect to the appropriateness of PAC use in CABG and AAR. The mean appropriateness score for PAC use in low-risk AAR was 3.19, suggesting substantial consensus regarding the inappropriateness of PAC use in this population. By contrast, the corresponding score for low-risk CABG patients was 4.25. Thus, among the respondents surveyed, a state of equipoise appears to exist regarding the appropriateness of PAC use in low-risk CABG. This finding is consistent with the recommendations of the PACCO workshop and the ASA Task Force. ${ }^{16,17}$ Third, the range for equipoise defined by Jacka et al. is rather narrow (mean scores of 4.0-6.0) and encompasses only two units in a range of eight (1.0-9.0). It might be more appropriate to have equipoise encompass one third of this range (3.7-6.3). This would be consistent with the scoring system used by individual respondents, where 3 of 9 possible scores were to be regarded as indicating uncertainty. If the range for equipoise were slightly expanded in this manner, it would encompass the scores for the "usual patient" with stable angina and good LV function undergoing either CABG or AAR (mean score 3.81) as well as those for patients with unstable angina and good LV function 
(mean score 6.25) undergoing either procedure. Fourth, the appropriateness scores may not reflect the frequency of PAC use in patients undergoing CABG or AAR. In their multivariable analysis, Jacka et al. found that clinicians with the highest case volume also rated PAC use as more appropriate. So, the number of patients who are actually instrumented with PACs is likely higher than indicated by the appropriateness scores. Thus, a more pragmatic definition of equipoise, where $30-70 \%$ of patients actually undergo PA catheterization perioperatively, may be fulfilled for low-risk patients undergoing CABG or AAR. Finally, it may be ethical to perform a RCT when appropriateness scores fall outside the range of equipoise, if clinicians recognize that their scores are based on weak evidence. This is likely the case regarding PAC utilization in cardiovascular surgery.

In summary, Jacka et al. have done us a service by helping to define clinicians' attitudes regarding the appropriateness of PAC use. Meanwhile, cardiovascular anesthesiologists continue to make decisions regarding PAC use in the absence of high-quality evidence regarding efficacy. The PAC continues to be widely employed in low-risk patients undergoing high frequency procedures like CABG and AAR. ${ }^{4}$ Because PAC use is not without cost or risk, well-designed RCTs conducted in these populations would be extremely helpful. The essential characteristics of a meaningful RCT have been well described. ${ }^{18}$ These include a large sample size, protocol-guided treatment responses to PAC-derived data, elimination of crossover from CVP to PAC groups, and a focus on clinically relevant outcomes. The survey by Jacka $\mathrm{et} a \mathrm{l}$. suggests to me that it would be ethical to conduct a multicentre RCT examining the utility of PAC use in patients without severe LV dysfunction, with or without unstable angina, undergoing CABG. I believe such a study would also be feasible, and fundable.

\section{Le cathéter artériel pul- monaire en chirurgie cardio-vasculaire}

Depuis l'introduction, en 1970, du cathéter à extrémité gonflable, le cathéter artériel pulmonaire (CAP) a été largement utilisé chez les patients de chirurgie cardio-vasculaire. Les anesthésiologistes ont d'abord adopté le CAP avec enthousiasme et beaucoup l'ont utilisé pour des pontages aortocoronariens (PAC) ou la reconstruction de l'aorte abdominale (RAA). Ils ont cru qu'en mesurant des variables qui ne sont pas accessibles à l'évaluation clinique courante (pression artérielle pulmonaire bloquée, saturation en oxygène du sang veineux mêlé), la démarche périopératoire allait changer et améliorer le devenir du patient. Cependant, l'ardeur qui a pu exister entre les anesthésiologistes cardio-vasculaires et le CAP, quoique prolongée et intense, semble maintenant se refroidir. Cette impression est confirmée par les résultats d'une enquête menée par Jacka et coll. et publiés dans le présent numéro du Journal. ${ }^{1}$

Jacka et coll. ont sondé 345 anesthésiologistes de 29 centres au Canada et aux États-Unis pendant les quatre premiers mois de 1998 . Des 265 répondants $(76,8 \%)$, 214 offraient de l'anesthésie au moins une fois par mois en chirurgie cardiaque ou vasculaire. Les réponses de ces 214 "anesthésiologistes cardio-vasculaires" font l'objet de la présente publication. On a demandé aux répondants d'évaluer la pertinence de l'usage du CAP pour différents scénarios cliniques portant sur des malades qui subissent un PAC ou une RAA non urgents. La pertinence a été estimée par une échelle en neuf points allant de 1 , complètement non pertinent, à 9 , complètement pertinent. Les réponses ont été groupées comme suit : non pertinent $(1-3)$, incertain $(4-6)$ et pertinent $(7-9)$. Seulement $19 \%$ des répondants l'ont jugé pertinent pour le malade habituel opéré pour un PAC ou un RAA et dont l'angine est stable et la fonction ventriculaire normale. Toutefois, on s'entendait sur l'utilisation pertinente du CAP dans certains scénarios à haut risque. Le CAP a aussi été jugé pertinent, par $74 \%$ des répondants, pour les malades porteurs de dysfonction (fraction d'éjection $\mathrm{VG}<0,4)$ du ventricule gauche (VG) et 87 $\%$ l'ont dit pertinent dans les cas combinant une angine instable et une atteinte VG fonctionnelle.

On ne saurait dire exactement pourquoi les anesthésiologistes cardio-vasculaires sont maintenant déçus de l'usage courant du CAP. L'explication la plus plausible serait qu'après 30 ans d'expérience, les grands essais randomisés et contrôlés (ERC) n'indiquent plus d'amélioration évidente reliée à l'usage du CAP chez les malades de chirurgie cardiovasculaire. Chez ceux qui subissent un PAC, la meilleure preuve disponible indique que l'usage du CAP ne modifie pas les résultats, mais pourrait augmenter l'utilisation des ressources. ${ }^{2}$ Lors d'une étude rétrospective, on a observé que les patients à faible risque d'un PAC, comportant seulement le monitorage de la pression veineuse centrale (PVC), connais- 
sent une bonne récupération. ${ }^{3}$ Une importante étude rétrospective de cohortes a montré l'usage du CAP associé à une hausse de la mortalité et à l'augmentation d'utilisation des ressources dans les cas de PAC. ${ }^{4}$ Chez les malades à faible risque d'une RAA, trois ERC à faible échantillonnage indiquent aucune amélioration des résultats associée au CAP comparé au monitorage de la PVC. ${ }^{5-7}$ Dans deux de ces études, ${ }^{5,6}$ aucun protocole d'utilisation des données du CAP pour la thérapie n'a été fourni, tandis que dans le troisième essai, le CAP a été utilisé pour orienter le traitement voulant atteindre des objectifs hémodynamiques spécifiques. ${ }^{7}$ Dans deux autres petits ERC, des cas d'intervention vasculaire non urgente (certains cas de RAA), le CAP a guidé le traitement visé qui était "d'optimaliser" la distribution d'oxygène. ${ }^{8,9}$ Ces études ont aussi montré aucune amélioration des résultats associée au CAP. Les anesthésiologistes cardio-vasculaires ont pu être influencés aussi par les résultats d'études par observation, non contrôlées, dans lesquelles l'usage du CAP a été associé à une hausse de la mortalité et de l'utilisation des ressources chez différentes populations de malades, y compris les cas d'infarctus myocardique compliqué, ${ }^{10}$ de maladie grave $^{11}$ ou d'opération non cardiaque. ${ }^{12}$ Dans la plupart de ces dernières publications, le risque de mortalité accrue reliée à l'usage du CAP dans une population mixte de malades traités dans des unités de soins intensifs, était plus grand à la suite d'une opération importante. ${ }^{11}$ Aussi, les limites financières ont pu forcer les médecins à faire un usage plus rentable du matériel, entraînant en cela une baisse accélérée de l'usage de CAP. L'expérience clinique de bons résultats obtenus sans monitorage avec le CAP peut avoir contribué à cette attitude. Enfin, des techniques concurrentes comme l'échographie transœsophagienne (ETO) pourraient évincer l'usage du CAP en clinique. Tout en étant moins effractive, l'ETO fournit plus d'informations objectives sur la précharge et la fonction ventriculaire que le monitorage du CAP. ${ }^{13}$ Jacka et coll. ont sans doute considéré aussi cette dernière explication, puisqu'ils ont fait parvenir des questionnaires à des anesthésiologistes d'établissements choisis "d'après les différences connues d'accessibilité à l'ETO." Il est dommage que les résultats ne mentionnent pas l'effet de l'accessibilité à l'ETO sur la cote de pertinence.

L'article de Jacka et coll. est intéressant pour un certain nombre de raisons. Premièrement, en l'absence d'arguments de qualité, les opinions d'un confrère peuvent constituer un guide utile de bonne pratique. Deuxièmement, les résultats de l'enquête peuvent aussi indiquer des domaines pertinents pour de futures recherches. Ainsi, on a proposé que les essais cliniques soient justifiés quand un état "d'équipoise clinique" existe. ${ }^{14} \mathrm{Il}$ y a équipoise quand un pourcentage significatif de cliniciens, habituellement $30-70 \%$, est incertain de l'utilité potentielle d'une thérapie ou d'un appareil spécifique. Quand il n'y a pas d'équipoise, les essais cliniques peuvent être contraires à l'éthique. À ce sujet, Jacka et coll. avancent que les essais cliniques sur l'utilité du CAP pourraient manquer à l'éthique chez le malade habituel, qui présente de l'angine stable et une fraction d'éjection VG 0,4 , opéré pour un PAC ou une RAA, parce que leur enquête évoque l'absence d'équipoise. L'estimation de la pertinence moyenne pour l'usage du CAP dans ce scénario a été de 3,81, tandis que la zone d'équipoise clinique employée par Jacka et coll. avait un score moyen de 4,0 $-6,0$. Des objections similaires peuvent être soulevées quant à la conduite d'essais cliniques auprès de malades qui présentent une dysfonction VG (score de pertinence moyen $=7,19$ ) .

Certains protocoles d'accord majeurs ont évalué l'avantage du CAP en chirurgie cardio-vasculaire et fait des recommandations sur la nécessité d'essais cliniques supplémentaires. ${ }^{15-17}$ Fait intéressant à noter, les opinions des experts de ces conférences ne vont pas uniformément dans le sens des constatations de Jacka et coll. Les participants à la Pulmonary Artery Catheter Consensus Conference ont conclu que le CAP n'est pas indiqué chez les malades à faible risque qui subissent un PAC, quoique la preuve de cette assertion ait été dotée d'une classe $\mathrm{C}$ (soutenue par de petits essais randomisés aux résultats incertains). ${ }^{15}$ Par contre, ces experts ont conclu que l'avantage du CAP était incertain chez les malades à haut risque subissant un PAC (déclaration fondée aussi sur une preuve de classe C) et ils recommandent des essais cliniques chez cette population. Récemment, l'atelier de Pulmonary Artery Catheterization and Clinical Outcomes (PACCO), sous la direction du National Heart, Lung and Blood Institute and the Food and Drug Administration, a reconnu "le PAC à faible risque" comme l'un des trois domaines de grande importance pour de futurs essais cliniques contrôlés et randomisés sur l'utilité du CAP. ${ }^{16}$ Finalement, l'American Society of Anesthesiologists (ASA) Task Force sur le cathétérisme artériel pulmonaire a conclu que "les futurs essais randomisés et contrôlés [sur l'utilité du $\mathrm{CAP}]$ devraient porter sur des malades à faible risque (c.-à-d., une fraction d'éjection plus grande que $50 \%$ ) et restreindre l'analyse à un type d'intervention spécifique (comme le PAC)". ${ }^{17}$ Ces conclusions disparates présentées par les différents groupes d'experts et l'enquête de Jacka et coll., montrent toute la controverse 
et le manque de preuves sur l'utilité du CAP en chirurgie cardio-vasculaire.

La preuve apportée par Jacka et coll. nous incite-telle à abandonner, comme contraire à l'éthique, l'idée de mener de grands essais randomisés et contrôlés sur l'utilité du CAP chez les malades à faible risque en chirurgie cardio-vasculaire? Nous croyons que non. D'abord, il y a eu peu de répondants à l'enquête et les résultats ne traduisent peut-être pas exactement l'opinion de tous les anesthésiologistes cardio-vasculaires nord américains. D'après la clientèle rapportée, on peut penser que certains répondants étaient relativement inexpérimentés en anesthésie cardio-vasculaire. Deuxièmement, les répondants ne sont pas tous d'accord sur la pertinence du CAP pour un PAC ou une RAA. Le score de pertinence moyen pour le CAP chez les malades en RAA à faible risque était de 3,19, ce qui suppose un consensus important sur la non pertinence du CAP dans cette population. En comparaison, le score correspondant pour le $\mathrm{PAC}$ à faible risque était de 4,25. Par conséquent, une équipoise semble exister sur la pertinence du CAP pour le PAC à faible risque. Cette constatation suit les recommandations de l'atelier PACCO et du groupe de travail de l'ASA. ${ }^{16,17}$ Troisièmement, les limites de l'équipoise définies par Jacka et coll. sont plutôt étroites (scores moyens de 4,0-6,0), avec seulement deux unités sur un intervalle de huit $(1,0-9,0)$. Une équipoise qui englobe un tiers de ces unités $(3,7-6,3)$ pourrait être plus appropriée. Cette situation cadrerait avec le système de cotation utilisé par des répondants particuliers, où 3 des 9 scores possibles ont été vus comme des indicateurs d'incertitude. Si les limites de l'équipoise étaient légèrement étendues ainsi, elles comprendraient les scores pour le "malade habituel", à l'angine stable et ayant une bonne fonction VG, opéré pour un PAC ou une RAA (score moyen de 3,81 ), autant que pour le malade qui a de l'angine instable et une bonne fonction VG (score moyen de $6,25)$ et qui subit l'une ou l'autre intervention. Quatrièmement, on peut douter de la correspondance entre les scores de pertinence et la fréquence d'utilisation du CAP dans les cas de PAC ou de RAA. Dans leur analyse multivariée, Jacka et coll. ont découvert que les cliniciens qui ont la clientèle la plus nombreuse estiment également l'usage du CAP comme plus approprié. Donc, le nombre de malades chez qui on utilise effectivement le CAP est probablement plus élevé que ce que laissent voir les scores de pertinence. Une définition plus pragmatique de l'équipoise, où 30 - $70 \%$ des malades subissent réellement un cathétérisme AP périopératoire, devrait donc être produite pour les malades à faible risque opérés pour un
PAC ou une RAA. Finalement, ce serait faire preuve d'éthique que de réaliser un ERC lorsque les scores de pertinence débordent les limites d'équipoise, si les cliniciens reconnaissent que leurs scores sont fondés sur une preuve peu concluante. C'est le cas, semble-til, de l'utilisation du CAP en chirurgie cardio-vasculaire.

En résumé, Jacka et coll. nous rendent service en contribuant à définir les attitudes des cliniciens sur la pertinence de l'usage du CAP. Entre-temps, les anesthésistes cardio-vasculaires décident encore de l'usage du CAP en l'absence d'une preuve solide de leur efficacité. Le CAP est toujours largement employé chez les malades à faible risque opérés pour un PAC ou une RAA, interventions pratiquées fréquemment. ${ }^{4}$ Comme l'usage du CAP comporte un coût et des risques, il est extrêmement souhaitable que des ERC bien conçus soient menés auprès de ces populations. Les caractéristiques essentielles d'un ERC significatif sont connues. ${ }^{18}$ Elles incluent un grand échantillon, des actions thérapeutiques dérivées des données du CAP et guidées par un protocole, l'élimination de croisement des groupes PVC aux groupes CAP et une focalisation sur les résultats cliniques utiles. L'enquête de Jacka et coll. donne à penser qu'un ERC multicentrique serait une manière éthique de vérifier l'utilité du CAP chez les malades sans dysfonction sévère du VG, avec ou sans angine instable, qui subissent un PAC. Nous croyons ce projet faisable et finançable.

\section{References}

I Jacka MJ, Cohen MM, To T, Devitt JH, Byrick R The appropriateness of the pulmonary artery catheter in cardiovascular surgery. Can J Anesth 2002; 49: 276-81.

2 Tuman KJ, McCarthy RJ, Spiess BD, et al. Effect of pulmonary artery catheterization on outcome in patients undergoing coronary artery surgery. Anesthesiology 1989; 70: 199-206.

3 Bashein G, Johnson PW, Davis KB, Ivey TD. Elective coronary bypass surgery without pulmonary artery catheter monitoring. Anesthesiology 1985; 63: 451-4.

4 Ramsey SD, Saint S, Sullivan SD, Dey L, Kelley K, Bowdle A Clinical and economic effects of pulmonary artery catheterization in nonemergent coronary artery bypass graft surgery. J Cardiothorac Vasc Anesth 2000; 14: 113-8.

5 Isaacson IJ, Lowdon JD, Berry AJ, et al. The value of pulmonary artery and central venous monitoring in patients undergoing abdominal aortic reconstructive surgery: a comparative study of two selected, random ized groups. J Vasc Surg 1990; 12: 754-60.

6 Joyce WP, Provan JL, Ameli FM, McEwan MM, Jelenich $S$, Jones DP. The role of central haemodynamic moni- 
toring in abdominal aortic surgery. A prospective randomised study. Eur J Vasc Surg 1990; 4: 633-6.

7 Valentine RJ, Duke ML, Inman MH, et al. Effectiveness of pulmonary artery catheters in aortic surgery: a randomized trial. J Vasc Surg 1998; 27 : 203-12.

8 Bender JS, Smith-Meek MA, Jones CE. Routine pulmonary artery catheterization does not reduce morbidity and mortality of elective vascular surgery. Results of a prospective, randomized trial. Ann Surg 1997; 226: 229-37.

9 Ziegler DW, Wright JG, Choban PS, Flancbaum L. A prospective randomized trial of preoperative "optimization" of cardiac function in patients undergoing elective peripheral vascular surgery. Surgery 1997; 122: 584-92.

10 Gore JM, Goldberg RJ, Spodick DH, Alpert JS, Dalen $J E$. A community-wide assessment of the use of pulmonary artery catheters in patients with acute myocardial infarction. Chest 1987; 92: 721-7.

11 Connors AF Jr, Speroff T, Dawson NV, et al., for the SUPPORT Investigators. The effectiveness of right heart catheterization in the initial care of critically ill patients. JAMA 1996; 276: 889-97.

12 Polanczyk CA, Robde LE, Goldman L, et al. Right heart catheterization and cardiac complications in patients undergoing noncardiac surgery. An observational study. JAMA 2001; 286: 309-14.

13 Benjamin E, Griffin K, Leibowitz AB, et al. Goaldirected transesophageal echocardiography performed by intensivists to assess left ventricular function: comparison with pulmonary artery catheterization. J Cardiothorac Vasc Anesth 1998; 12: 10-5.

14 Freedman B. Equipoise and the ethics of clinical research. N Engl J Med 1987; 317: 141-5.

15 Pulmonary Artery Catheter Consensus Conference Participants'. Pulmonary artery catheter consensus conference: consensus statement. Crit Care Med 1997; 25: 910-25.

16 Bernard GR, Sopko G, Cerra F, et al. Pulmonary artery catheterization and clinical outcomes: National Heart, Lung, and Blood Institute and Food and Drug Administration Workshop Report. JAMA 2000; 283: 2568-72.

17 American Society of Anesthesiologists Task Force on Pulmonary Artery Catheterization. Practice guidelines for pulmonary artery catheterization. A report by the American Society of Anesthesiologists Task Force on pulmonary artery catheterization. Anesthesiology 1993; 78: 380-94.

18 Tuman KJ, Roizen MF. Outcome assessment and pulmonary artery catheterization: why does the debate continue? (Editorial). Anesth Analg 1997; 84: 1-4. 